\title{
Restoring MLL reactivates latent tumor suppression-mediated vulnerability to proteasome inhibitors
}

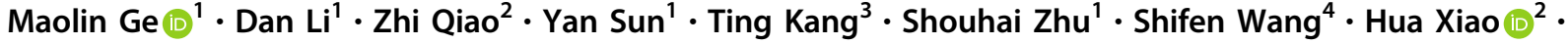 \\ Chunjun Zhao ${ }^{1}$. Shuhong Shen $\mathbb{1}^{5} \cdot$ Zhenshu Xu $\mathbb{1}^{4} \cdot$ Han Liu $\mathbb{1}^{1}$
}

Received: 12 March 2020 / Revised: 16 July 2020 / Accepted: 23 July 2020 / Published online: 30 July 2020

(c) The Author(s) 2020. This article is published with open access

\begin{abstract}
MLL undergoes multiple distinct chromosomal translocations to yield aggressive leukemia with dismal outcomes. Besides their well-established role in leukemogenesis, MLL fusions also possess latent tumor-suppressive activity, which can be exploited as effective cancer treatment strategies using pharmacological means such as proteasome inhibitors (PIs). Here, using MLL-rearranged xenografts and MLL leukemic cells as models, we show that wild-type MLL is indispensable for the latent tumor-suppressive activity of MLL fusions. MLL dysfunction, shown as loss of the chromatin accumulation and subsequent degradation of MLL, compromises the latent tumor suppression of MLL-AF4 and is instrumental for the acquired PI resistance. Mechanistically, MLL dysfunction is caused by chronic PI treatment-induced epigenetic reprogramming through the H2Bub-ASH2L-MLL axis and can be specifically restored by histone deacetylase (HDAC) inhibitors, which induce histone acetylation and recruits MLL on chromatin to promote cell cycle gene expression. Our findings not only demonstrate the mechanism underlying the inevitable acquisition of PI resistance in MLL leukemic cells, but also illustrate that preventing the emergence of PI-resistant cells constitutes a novel rationale for combination therapy with PIs and HDAC inhibitors in MLL leukemias.
\end{abstract}

\section{Introduction}

The mixed-lineage leukemia $(M L L)$ gene encodes a histone methyltransferase governing histone $\mathrm{H} 3$ lysine residue 4

These authors contributed equally: Maolin Ge, Dan Li, Zhi Qiao, Yan Sun

Supplementary information The online version of this article (https:// doi.org/10.1038/s41388-020-01408-7) contains supplementary material, which is available to authorized users.

Shuhong Shen

shenshuhong@scmc.com.cn

$\triangle$ Zhenshu Xu

zhenshuxu@yahoo.com

$\triangle$ Han Liu

liuhan68@sjtu.edu.cn

1 Shanghai Institute of Hematology, State Key Laboratory of Medical Genomics, National Research Center for Translational Medicine at Shanghai, Ruijin Hospital affiliated to Shanghai Jiao Tong University School of Medicine, 200025 Shanghai, China

2 State Key Laboratory of Microbial Metabolism, School of Life
(H3K4) methylation [1]. MLL orchestrates several essential cellular processes by positively regulating its target genes, especially the Hox gene family and cell cycle genes [2, 3]. MLL precursor polypeptide is site-specifically cleaved by the Taspase1 protease and functions as heterodimeric complexes composed of its amino (MLL ${ }^{\mathrm{N} 320}$ ) and carboxy $\left(\mathrm{MLL}^{\mathrm{C} 180}\right)$ terminal subunits $[4,5]$. The $M L L$ gene undergoes many distinct chromosomal rearrangements to yield aggressive acute lymphoblastic leukemia (ALL) and acute myeloid leukemia (AML). Leukemogenic MLL translocations fuse the N-terminal 1 1400 amino acids of MLL in

Sciences and Biotechnology, Shanghai Jiao Tong University, 200240 Shanghai, China

3 Department of Oncology, Xin Hua Hospital, School of Medicine, Shanghai Jiao Tong University, 200092 Shanghai, China

4 Fujian Institute of Hematology, Fujian Provincial Key Laboratory of Hematology, Fujian Medical University Union Hospital, 350001 Fuzhou, China

5 Key Laboratory of Pediatric Hematology and Oncology Ministry of Health, Department of Hematology \& Oncology, Pediatric Translational Medicine Institute, Shanghai Children's Medical Center, School of Medicine, Shanghai Jiao Tong University, 200127 Shanghai, China 
frame with more than 94 translocation partner genes, which are present at high frequency in infants and at lower frequencies in children and adults $[5,6]$.

In contrast to the rearranged allele, the other $M L L$ allele usually remains intact and expressed. The contribution of this wild-type MLL allele to leukemogenesis in MLLrearranged leukemias has been the subject of intense research. Several lines of investigation support that endogenous MLL maintains the H3K4me status and facilitates MLL-fusion protein-mediated leukemogenesis [7-9]. Meanwhile, the loss of endogenous MLL alone can have significant impacts on several AML subtypes, including those initiated by MN1 and NUP98 fusion proteins [10, 11]. However, other studies have demonstrated that endogenous MLL is dispensable for MLL-rearranged AML and that MLL deletion alone had no major impact on the survival of MLL leukemic cells [12, 13]. Nevertheless, these discrepancies occur mainly in AML models, while the contribution of the wild-type allele of MLL to MLL-rearranged ALL remains elusive.

The improved molecular understanding of MLL and MLL fusions has led to the identification of several potential mechanism-based therapeutic targets. While the necessity of the wild-type allele of MLL for leukemogenesis is debatable, it has nonetheless become an attractive therapeutic target in MLL leukemia. Given the findings that the remaining wild-type MLL protein is generally much less abundant than the MLL fusions in MLL leukemia cells, several candidate therapeutic strategies are emerging that stabilize wild-type MLL protein to displace MLL chimeras from chromatin and therefore evade the oncogenic addiction of these cells to MLL chimeras [14, 15]. For example, the inhibition of interleukin-1 receptor-associated kinases (IRAKs) impedes UBE2O-mediated MLL degradation and stabilizes wild-type MLL protein. Casein kinase II (CKII) inhibition, on the other hand, blocks the phosphorylation of the taspase1 cleavage site on MLL and inhibits taspase1dependent MLL processing, thus increasing MLL stability. Analogously, IRAK and CKII inhibition induce wild-type MLL to outcompete the oncogenic MLL chimeras through additional chromatin-binding modules, such as PHD fingers and a bromodomain. These domains are not retained in MLL fusions but exist exclusively in wild-type MLL [16]. Histone deacetylase (HDAC) inhibitors have also been reported to activate wild-type MLL [17], but the underlying mechanisms are not fully understood.

Proteasome inhibitors (PIs) are newly reported clinical regimens for MLL therapy, specifically MLL-r B-ALL cells, but not AML $[18,19]$. Mechanistically, proteasome inhibition induces the intrinsic tumor-suppressive activity of MLL fusions by triggering apoptosis and cell cycle arrest involving cleavage of BID by caspase- 8 and upregulation of $\mathrm{p} 27$, respectively $[18,20]$. The accumulation of endogenous MLL-fusion proteins at the $\mathrm{p} 27$ locus through PAX5 is decisive to the specific cytotoxicity caused by proteasome inhibition in lymphoid, but not myeloid, MLL leukemias.

We previously reported that PI bortezomib single-agent therapy showed effectiveness in mouse models and patients with pro-B MLL leukemia; however, the inevitable emergence of PI resistance imposes limits on bortezomib's clinical application [18]. Therefore, identification of the mechanism underlying PI resistance and the design of novel combination strategies are essential to overcome resistance and facilitate the application of PIs to MLL leukemias. Intriguingly, we found that the wild-type MLL protein was less abundant and was insensitive to PI treatment in resistant MLL leukemia cells, compromised the latent tumor suppression of MLL fusions. Therefore, we reasoned that disruption of the balance between wild-type MLL and MLL chimeras plays a critical role in PI resistance, and that targeting MLL dysfunction and restoring MLL may be a promising strategy for treating the aggressive resistance in MLL leukemia.

\section{Results}

\section{PI resistance is associated with the failure of MLL and p27 induction}

We previously observed that pro-B MLL leukemia displayed selective sensitivity to the PI bortezomib, but the disease did eventually develop in xenograft mice [18]. To understand mechanisms by which pro-B MLL leukemic cells overcome proteasome inhibition, we modeled bortezomib-resistant SEM and RS4;11 cells by continuously treated parental cells with a sublethal dose of bortezomib $(5 \mathrm{nM})$ for over 4 weeks (Figs. 1a and S1A). Cells were passaged and the inhibitor was replenished every 3 days. The half-maximal dosage effect $\left(\mathrm{IC}_{50}\right)$ values were measured intermittently until the drug resistance was acquired. In PI-sensitive MLL leukemic cells, bortezomib induces the accumulation of MLL-AF4 and latent tumor suppression programs, triggering cell cycle arrest involving the activation of the p27 gene $(C D K N 1 B)$ [18]. Like the original PI-sensitive parental cells, the abundance of MLLAF4 in resistant cells exhibited a remarkable increase under bortezomib treatment, while the abundance of wild-type MLL and p27 did not increase significantly (Fig. 1b), indicating that PI resistance in MLL leukemic cells is associated with the failure of MLL and p27 induction. Interestingly, while the activation of p27 is essential for cell cycle deregulation caused by acute PI treatment, it was not significantly activated in chronically induced PI-resistant cells (Fig. 1b), suggesting that the cell cycle deregulation in 
A

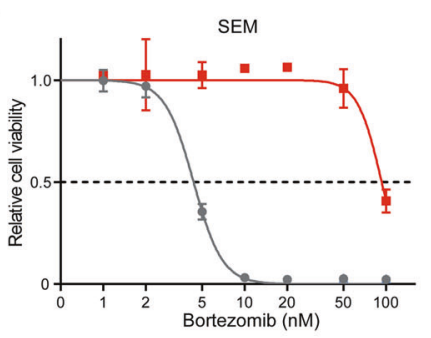

B

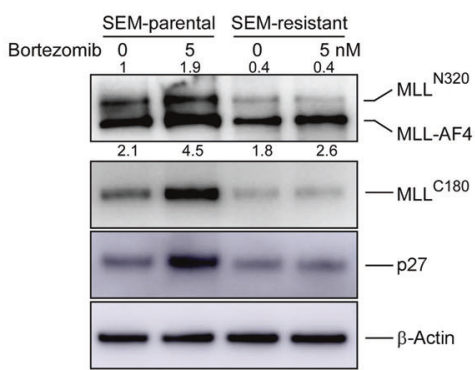

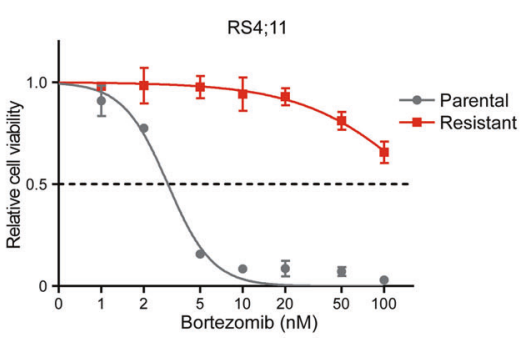

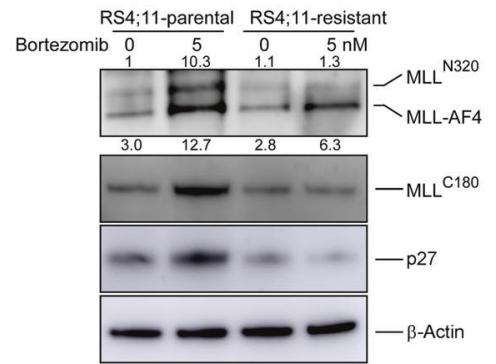

C

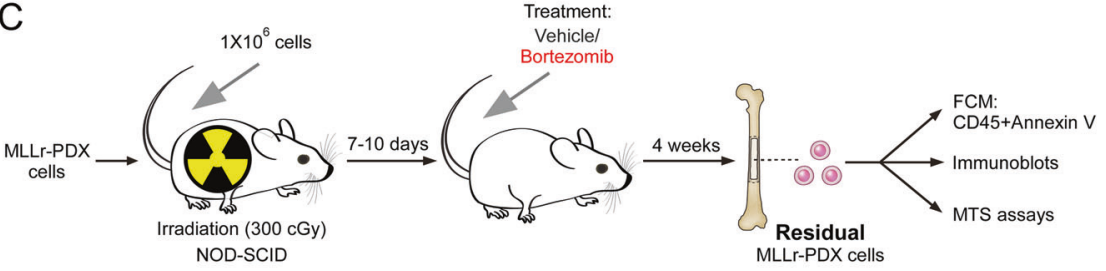

$\mathrm{F}$
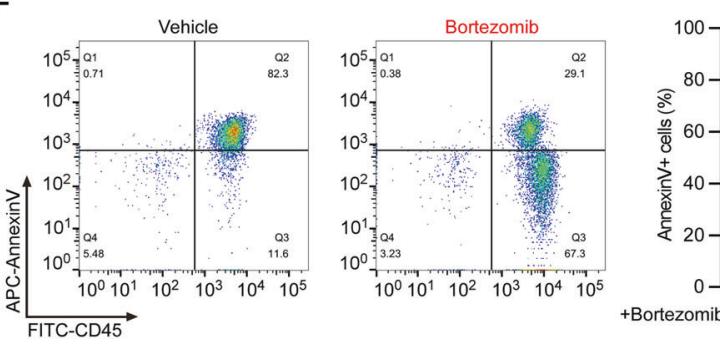

MLLr-PDX

Fig. 1 PI resistance is associated with the failure of MLL and p27 induction. a Cell viability of parental and resistant SEM and RS4;11 cells was measured with MTS assay $24 \mathrm{~h}$ after the addition of bortezomib at the indicated concentrations. b Immunoblots of the indicated cells before and after a $12 \mathrm{~h}$ exposure to bortezomib. The band intensity represents fold changes to internal control by using Image $\mathbf{J}$ software analysis. The results are shown as fold induction of MLL $^{\mathrm{N} 320}$ and MLL-AF4 relative to endogenous wild-type MLL control. c Schematic for the different treatment strategies on the MLL-r PDX cells. d, e Cell viability of bone marrow cells derived from MLL-

PI-resistant cells was achieved through an alternative mechanism.

To determine if the failure of MLL and p27 induction was present in patient-derived cells, we generated MLLrearranged patient-derived xenografts (MLL-r PDX) from two MLL-fusioned B-ALL patients (Fig. S1B, C, Table S1). We collected the bone marrow cells from xenograft mice treated with bortezomib and evaluated the sensitivity of these cells to bortezomib (Fig. 1c). Compared with the represented as mean $\pm \mathrm{SD}$.
D

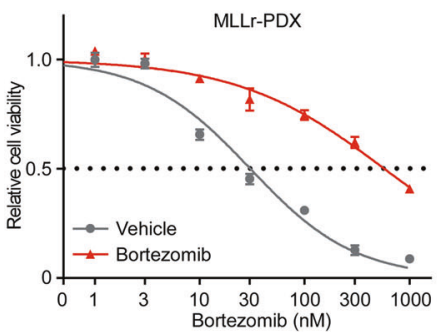

$E$

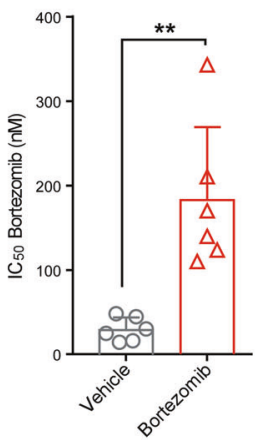

G

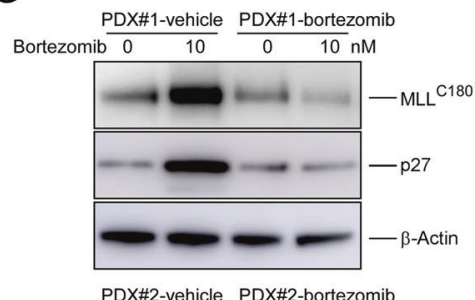

Bortezomib 0

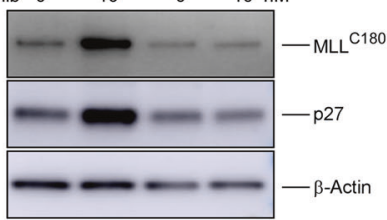

$\mathrm{r}$ PDX mice was measured with MTS assay $24 \mathrm{~h}$ after the addition of bortezomib. One representative of six independent experiments is shown in (d). Histogram of six independent biological replicates with three technical replicates is shown in (e). $\mathbf{f}$ Bone marrow cells from the MLL-r PDX mice were obtained and dead cells were removed. Flow cytometric detection of CD45 expression and apoptosis was measured $16 \mathrm{~h}$ after the addition of bortezomib. $\mathrm{g}$ Immunoblots of the indicated PDX cells before and after a $12 \mathrm{~h}$ exposure to the indicated concentrations of bortezomib. $* * P<0.01$; two-tailed $t$-test. Data are

xenograft mice treated with vehicle, cell viability of residual MLL-r PDX cells surviving bortezomib exposure was notably increased (Fig. 1d, e). We then examined the apoptosis of these residual cells, and the results showed that bortezomib treatment significantly reduced the apoptosis of bortezomib-treated MLL-r PDX cells, indicating that the residual MLL-r PDX cells surviving bortezomib exposure showed resistance to bortezomib (Fig. 1f). We further examined the expression of wild-type MLL and p27 
A

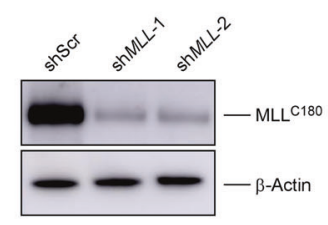

B

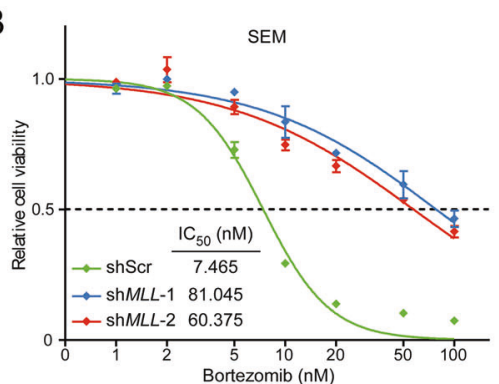

C

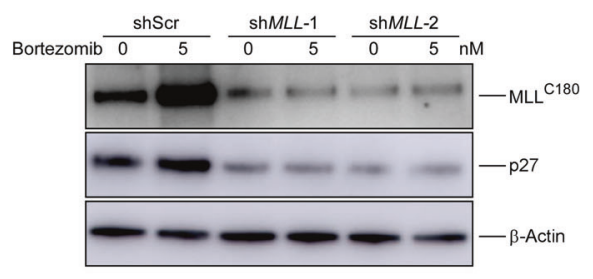

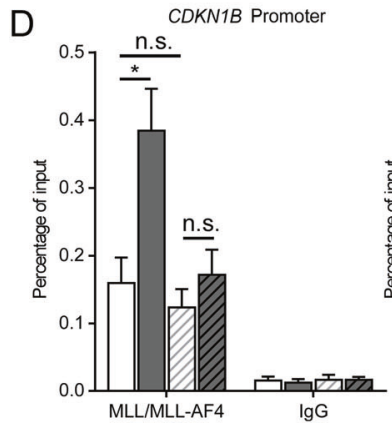
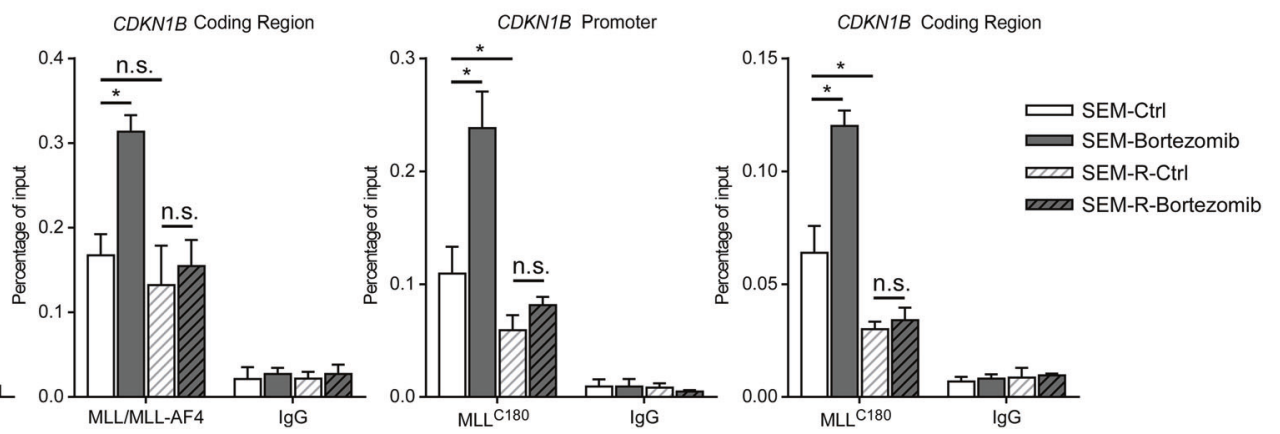

analyses at the promoter and coding region of the $C D K N 1 B$ locus in the indicated cells upon bortezomib treatment. MLL ${ }^{\mathrm{N} 320}$ (D2M7U) antibody against the shared amino terminus of MLL and MLL-AF4 was utilized. R resistant; n.s. not significant; $* P<0.05$; two-tailed $t$ test. Data are represented as mean $\pm \mathrm{SD}$. tified. c Immunoblots of SEM cells infected by the indicated lentiviral vectors before and after a $12 \mathrm{~h}$ exposure to bortezomib. d ChIP

proteins. Compared with the xenograft mice treated with vehicle, the induction of wild-type MLL and p27 was not significant in the bortezomib-treated MLL-r PDX mice (Fig. $1 \mathrm{~g})$. Hence, these results indicate that PI resistance is associated with the failure of MLL and p27 to be activated and cause cell cycle arrest and subsequent apoptosis.

The transcriptional regulation of $\mathrm{p} 27$ by MLL and MLL fusions has been reported in various experimental settings $[18,21,22]$. To confirm the association of MLL suppression with PI sensitivity and p27 expression in MLL leukemia, we knocked down the MLL gene in SEM cells and observed that MLL depletion significantly decreased the sensitivity of SEM cells to bortezomib (Fig. 2a, b), reduced the expression of p27 and suppressed the induction of p27 by bortezomib (Fig. 2c). We further performed chromatin immunoprecipitation (ChIP) assays to examine the occupancy of MLL and MLL/MLL-AF4 at both the CDKNIB promoter and coding regions. In fact, in bortezomib-treated SEM cells, the binding of MLL/MLL-AF4 and MLL at both the $C D K N 1 B$ promoter and coding region was increased (Fig. 2d). However, bortezomib-induced accumulation of MLL/MLL-AF4 and MLL was abolished in PI-resistant cells (Fig. 2d). Moreover, compared to parental cells, PIresistant cells showed a significantly decreased level of $C D K N 1 B$ promoter- and coding region-bound MLL, but not that of MLL/MLL-AF4 (Fig. 2d). Taken together, our data favor a positive correlation between MLL and p27 expression, indicating that MLL dysfunction compromises the latent upregulation of p27 by MLL-AF4 and is conducive to acquire PI resistance.

\section{The cell cycle of PI-resistant MLL leukemic cells is dysregulated}

MLL is involved in the positive regulation of cell cycle control $[2,3]$. To understand the effects of MLL dysfunction on cell cycle progression of PI-resistant cells, we examined the cell cycle and proliferation. As expected, the PI-resistant MLL leukemic cells SEM and RS4;11 were slow cycling with a low S-phase fraction (Figs. 3a and S2A), and cell proliferation was significantly suppressed (Figs. 3b and S2B). We subsequently performed RNA sequencing (RNA-Seq) to further characterize these PIresistant cells (Figs. 3c and S2C). Upon functional annotation clustering using DAVID, enrichment of genes involved in the mitotic cell cycle, G1/S transition, and negative regulation of cell proliferation was observed (Figs. $3 \mathrm{~d}$ and S2D). Using gene set enrichment analysis (GSEA), we established that genes associated with cell cycle processes were significantly enriched in both SEM and RS4;11 PI-resistant cells (Figs. 3e and S2E, Tables S2 and S3). Taken together, these results indicate that the cell cycle progression of PI-resistant cells mediated by MLL dysfunction is notably blocked. 
A
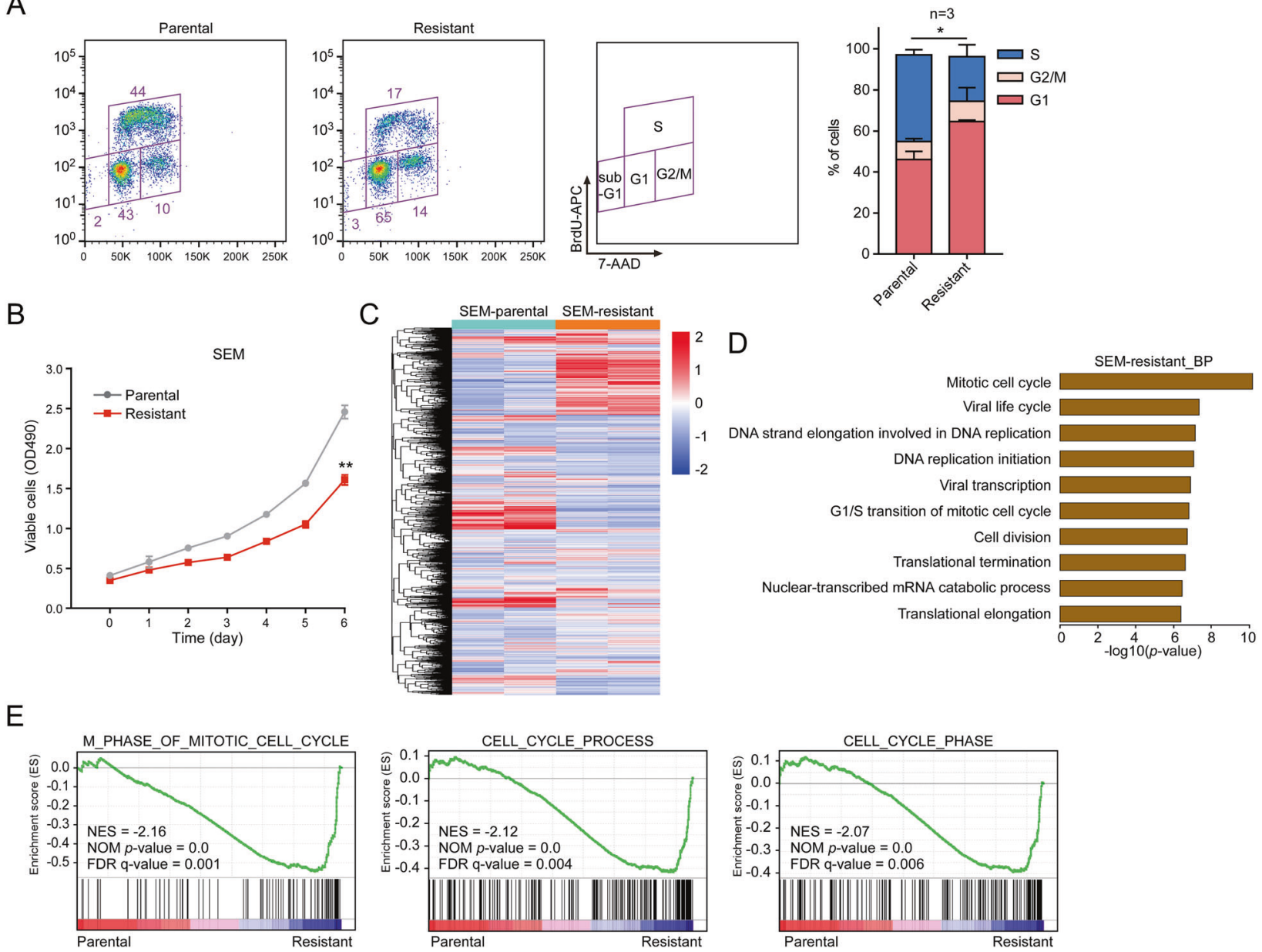

Fig. 3 The cell cycle of PI-resistant MLL leukemic cells is dysregulated. a Cell cycle profiling of SEM parental and resistant cells. Stacked barplot shows the fraction of cells viable in G1, S and G2/M phases of the indicated cells (three independent biological replicates with three technical replicates each). b The proliferation of SEM parental and resistant cells for 6 days. c Unsupervised hierarchical clustering heatmap of differentially expressed genes (DEGs) in SEM parental and resistant cells. d Biological process (BP) enrichment

\section{PI induces MLL dysfunction through epigenetic reprogramming}

Since $M L L$ mRNA was not significantly decreased (Fig. S3A) and MLL protein was less stable in PI-resistant cells (Fig. S3B), we examined whether PI resistance could be reversed by restoring the protein level of MLL using the IRAK1/4 inhibitor [14, 23]. Surprisingly, even though MLL protein was notably induced by IRAK1/4 inhibitor in PIresistant cells (Fig. S3C), the sensitivity to bortezomib could not be rescued (Fig. S3D). Interestingly, upon IRAK1/4 inhibitor treatment, while both chromatin-bound and free nucleoplasmic MLL were proportionally increased in the parental cells, only nucleoplasmic MLL was increased in the resistant cells (Fig. 4a). Consistent with this, less MLL was chromatin-bound in the resistant cells

analysis of DEG datasets obtained from SEM parental and resistant cells. GO analysis was performed with DAVID, and items were ordered by $P$ value. e The DEGs of SEM parental and resistant cells were studied with GSEA analysis. Normalized enrichment score (NES), nominal (NOM) $P$ value, and false discovery rate (FDR) are indicated. $* * P<0.01$; two-tailed $t$-test. Data represent the means of triplicate reactions $\pm \mathrm{SD}$.

(Fig. 4a). Since stabilized MLL could accumulate on chromatin to trimethylate $\mathrm{H} 3 \mathrm{~K} 4[3,14]$, our results that stabilized MLL failed to accumulate on chromatin suggested unfavorable chromatin accessibility for MLL in these resistant cells, which suppresses the effect of IRAK1/4 inhibitor on PI-resistant cells. Furthermore, chromatinassociated MLL was more stable than the nucleoplasmic MLL (Fig. S3E). Taken together, these results suggest that in PI-resistant cells, it is the unfavorable chromatin accumulation of MLL that caused less MLL to be recruited onto chromatin, resulting in the degradation and reduction of total MLL protein, but not vice versa.

We next investigated how the chromatin accumulation of MLL was reduced upon PI treatment. Consistent with previous reports, we observed that PI treatment depleted ubiquitinated histone H2B (H2Bub) [24] in RS4;11 and SEM 


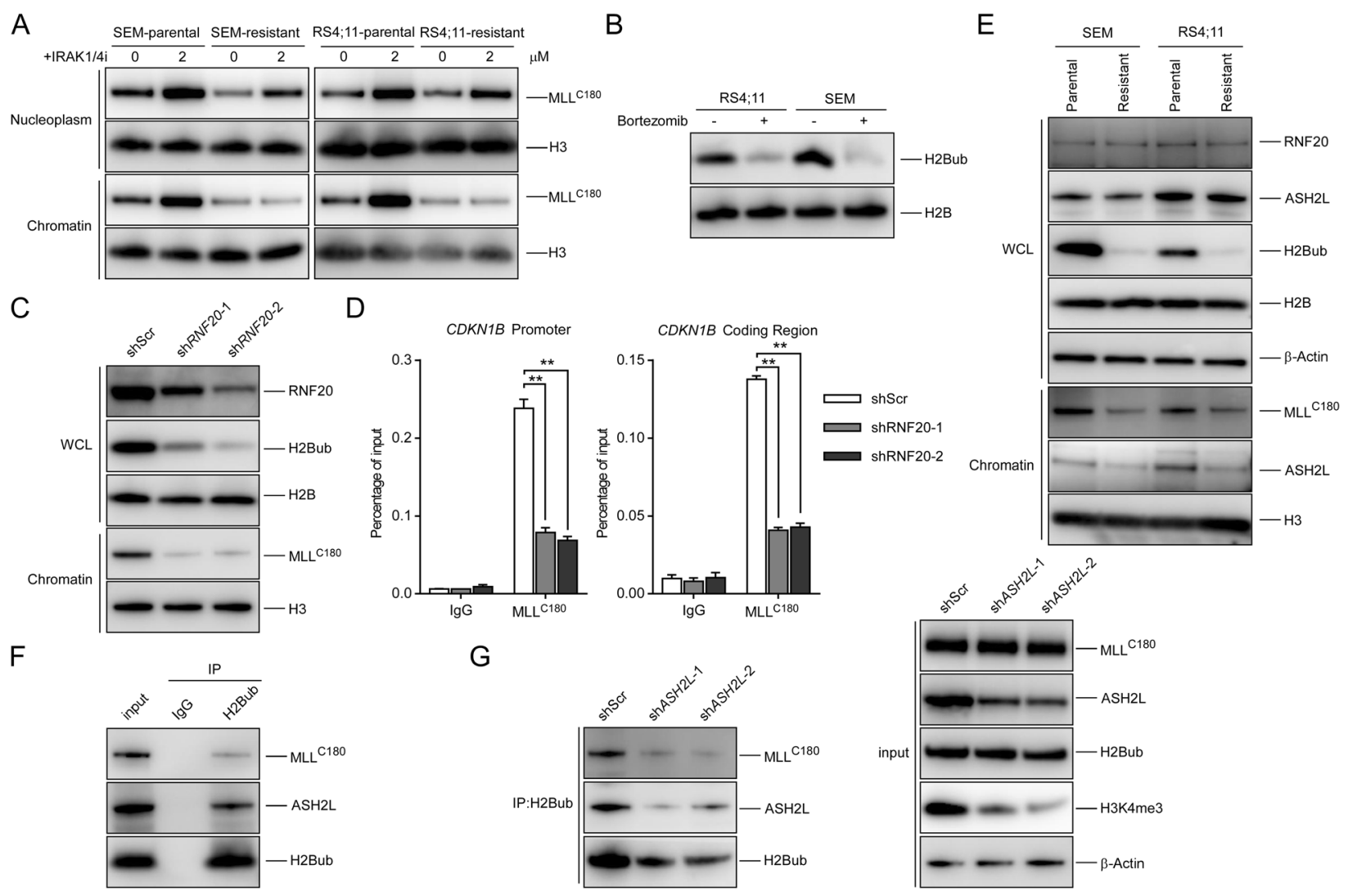

Fig. 4 PI induces MLL dysfunction through H2Bub depletion. a The indicated cells were treated with IRAK1/4 inhibitor for $48 \mathrm{~h}$. Nucleoplasm and chromatin-bound fractions were purified and immunoblots of the indicated antibodies were analyzed. b Immunoblots of H2Bub treated with bortezomib $(10 \mathrm{nM})$ for $8 \mathrm{~h}$ in the indicated cells. c SEM cells were infected by the indicated lentiviral vectors. ChIP analyses at the promoter and coding region of the $C D K N 1 B$ locus in the indicated cells were detected. d SEM cells were

cells (Fig. 4b). RNF20 is the major E3 ubiquitin ligase for histone H2B and RNF20 depletion causes a global reduction in H2Bub level [25]. We further examined whether the reduction in H2Bub caused by depleting RNF20 could impair the recruitment of MLL to chromatin. Indeed, upon RNF20 depletion and a reduction in H2Bub levels, the chromatin-bound MLL and CDKNIB locus-bound MLL were significantly decreased (Fig. 4c, d). We further observed that H2Bub in PI-resistant cells was reduced to lower levels than in parental cells, showing a good correlation with the amount of chromatin-bound MLL (Fig. 4e). However, there was no significant change in RNF20 expression, suggesting that the reduction in chromatinbound MLL is caused by PI treatment-induced depletion of $\mathrm{H} 2 \mathrm{Bub}$, rather than by RNF20 reduction.

Next, we addressed how H2Bub depletion could impair the recruitment of MLL to chromatin. It has been reported that ASH2L in the MLL complex-mediated H2Bubdependent H3K4 methylation [26-28]. Similar to chromatin-bound MLL, chromatin-bound ASH2L was also infected by the indicated lentiviral vectors. Immunoblots of WCL and chromatin-bound proteins were detected. e Immunoblots of whole-cell lysates (WCL) and chromatin-bound proteins in the indicated SEM or RS4;11 cells. f SEM cells subjected to anti-H2Bub immunoprecipitation (IP) and analyzed with the indicated antibodies. $\mathrm{g}$ SEM cells of the indicated knockdown were subjected to anti-H2Bub IP and analyzed with the indicated antibodies.

decreased in PI-resistant cells (Fig. 4e). Moreover, our coimmunoprecipitation assays confirmed that ASH2L and MLL could be recruited to H2Bub (Fig. 4f). We, therefore, hypothesized that PI treatment may decrease the $\mathrm{H} 2 \mathrm{Bub}$ level to prevent ASH2L-mediated recruitment of MLL to chromatin. To confirm the critical role of ASH2L in mediating the recruitment of MLL to H2Bub, we depleted ASH2L and observed that the recruitment of MLL to H2Bub was abolished (Fig. 4g). The results indicate that the depletion of $\mathrm{H} 2 \mathrm{Bub}$ induced by PI treatment decreases ASH2L-mediated recruitment of MLL to chromatin and causes the deregulation of a subset of cell cycleassociated genes.

\section{HDAC inhibitors restore cell sensitivity by enhancing the chromatin accessibility of MLL}

Our previous results showed that MLL fusions functioned as dominant-negative mutants by preventing the stabilization of wild-type MLL [3]. While the IRAK1/4 inhibitor can 
A

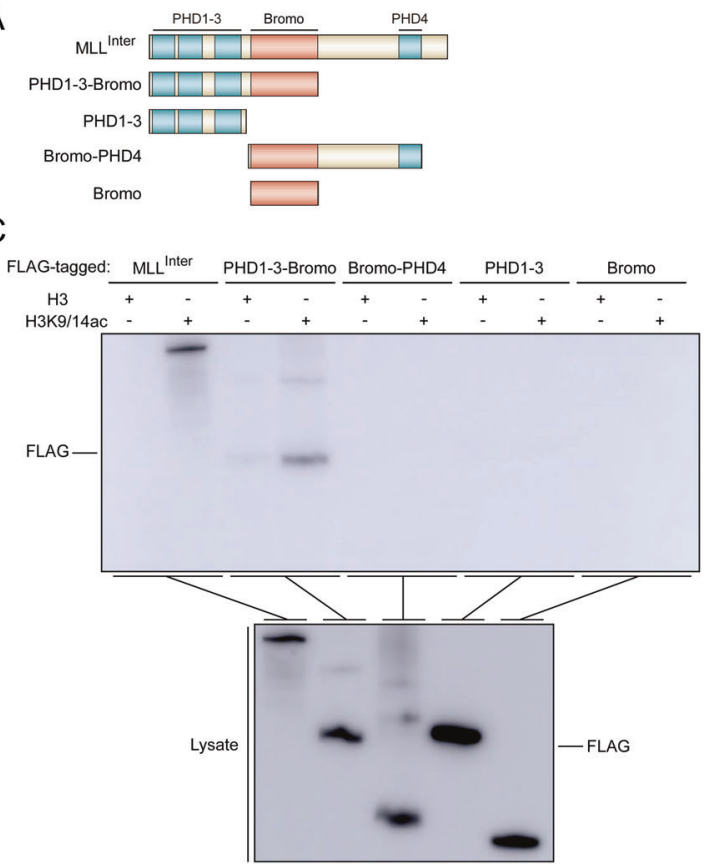

Fig. 5 MLL interacts with H3K9/14ac. a The construction of MLL domains. 293T cells were transfected with the indicated plasmids for $24 \mathrm{~h}$ and subjected to co-immunoprecipitation assays (b) or biotinconjugated histone tail pull-down assays (c). The co-precipitated

recruit stabilized MLL to chromatin and displace MLLfusion protein in 293T cells [14], it failed to do so in the resistant cells because of an epigenetic barrier (Fig. 4a). In order to develop an effective epigenetic therapy strategy that can tackle the resistance, we sought an epigenetic drug that could increase chromatin-bound MLL in the resistant cells. MLL possesses several putative chromatin targeting domains including three AT-hooks, one CXXC domain, four PHDs, and one bromodomain [29, 30]. Unlike the AThooks and CXXC domain, the PHDs and bromodomain are not retained in MLL fusions and exist exclusively in wildtype MLL [16]. Therefore, we focused on epigenetic changes that could specifically enhance chromatin accessibility to the PHDs and bromodomain, so that it would be clinically translatable to MLL leukemia.

It has been noted that wild-type MLL can be activated by class I HDAC inhibitors and displace MLL-AF4 from the promoter [17]. We thus reasoned that histone acetylation might facilitate chromatin accessibility for MLL. Bromodomains are well documented as acetyl-lysine-reading modules [31]. Although previous reports showed that MLL-Bromo was unable to recognize acetylated lysine residues after acetylation of $\mathrm{H} 4 \mathrm{~K} 12, \mathrm{H} 4 \mathrm{~K} 16, \mathrm{H} 3 \mathrm{~K} 23$, and H3K27 [30], our co-immunoprecipitation assays and peptide pull-down assays demonstrated that PHD1-3 along with Bromo could directly interact with $\mathrm{H} 3 \mathrm{~K} 9 / 14 \mathrm{ac}$ (Fig. 5a-c). We further confirmed that wild-type MLL, but not MLL-fusion proteins, interacted with H3K9/14ac (Fig.
B

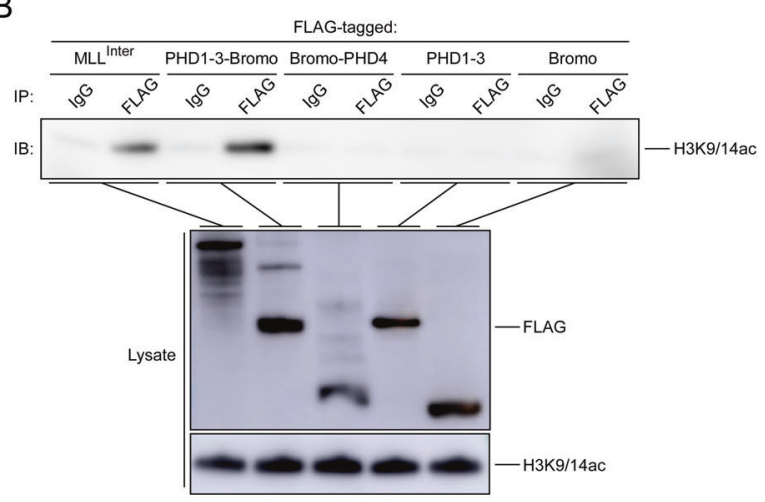

D

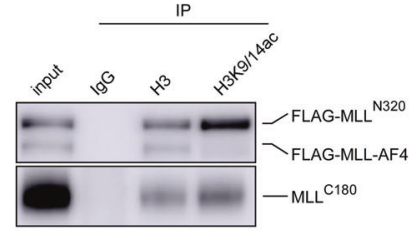

protein was detected by immunoblots. d $293 \mathrm{~T}$ cells co-transfected with the indicated plasmids for $24 \mathrm{~h}$ were subjected to coimmunoprecipitation assays. The co-precipitated protein was detected by immunoblots.

5d). In agreement with this, it has been reported that $\mathrm{H} 3 \mathrm{~K} 14 \mathrm{ac}$ is required for $\mathrm{H} 3 \mathrm{~K} 4$ trimethylation by COMPASS, an evolutionarily conserved histone methyltransferase complex containing an MLL ortholog [32]. Taking these results together, we hypothesized that the altered chromatin state using HDAC inhibitors in PI-resistant cells might overcome bortezomib resistance.

The HDAC inhibitor agents panobinostat (LBH589) and vorinostat (SAHA) are currently being tested in combination with various anticancer therapies [33-35]. Notably, when PIresistant leukemic cells were treated with LBH589 or SAHA, we observed an increase in $\mathrm{H} 3 \mathrm{~K} 9 / 14 \mathrm{ac}$, chromatin-associated MLL, and H3K4me3 expression (Fig. 6a). Moreover, HDAC inhibitors significantly induced $C D K N 1 B$ locus-bound $\mathrm{H} 3 \mathrm{~K} 9 /$ 14ac and MLL, correlated with an increase in p27 activation (Figs. 6b and S4A). In order to determine whether HDAC inhibitor-induced upregulation of p27 is MLL dependent, we knocked down the $M L L$ gene in PI-resistant SEM cells (Fig. S4B) and observed that MLL depletion significantly suppressed the induction of p27 by HDAC inhibitors (Fig. S4C). These data suggest that HDAC inhibitors lead to MLL accumulation on chromatin and directly promote the expression of the $C D K N 1 B$ gene and restore p27 induction by PIs in PI-resistant cells.

To investigate the therapeutic potency of HDAC inhibitors in PI-resistant cells, we examined the effect of HDAC inhibitors in combination with bortezomib. The results demonstrated that co-treatment of bortezomib with LBH589 


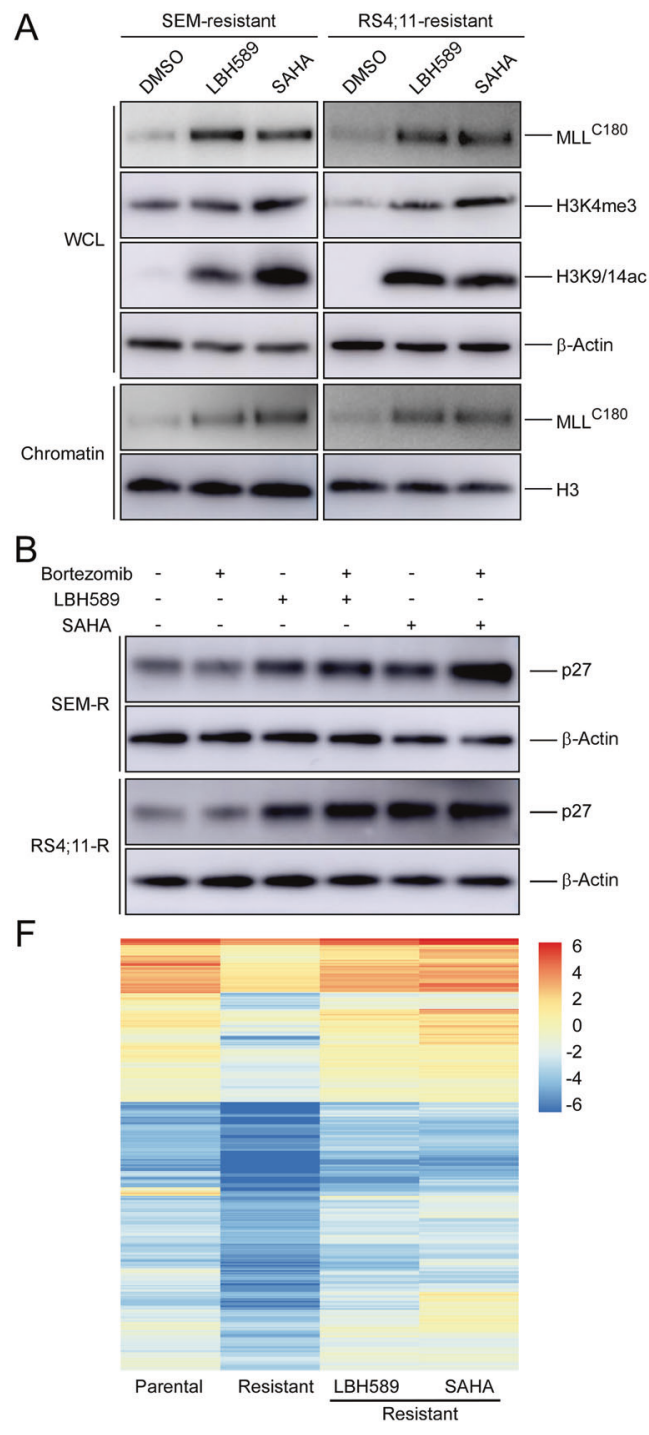

Fig. 6 HDAC inhibitors restore cell sensitivity by enhancing the chromatin accumulation of MLL. a SEM-resistant and RS4;11resistant cells were treated with DMSO, the HDAC inhibitor LBH589 ( $5 \mathrm{nM}$ for SEM and $50 \mathrm{nM}$ for RS4;11), or SAHA $(2 \mu \mathrm{M})$ for $20 \mathrm{~h}$. Immunoblots of the indicated antibodies were analyzed. b SEMresistant (SEM-R) and RS4;11-resistant (RS4;11-R) cells were treated with HDAC inhibitors described in (a), and then treated with $5 \mathrm{nM}$ bortezomib for $12 \mathrm{~h}$. Immunoblots of the indicated antibodies were analyzed. c The indicated cells were treated with DMSO or HDAC

or SAHA led to a strong reduction in cell viability (Fig. 6c), which was correlated with marked induction of cells in Sphase (Figs. 6d and S4D). Total RNA-seq revealed that in the presence of HDAC inhibitors, a large proportion of downregulated genes in SEM and RS4;11-resistant cells were notably induced (Figs. 6e, f and S4E, F, Tables S4 and S5). To determine the set of genes commonly deregulated in PI-resistant cells under HDAC inhibitor treatment, we analyzed the overlap of differentially expressed genes (DEGs) and gene sets. Venn diagram analysis identified 1959 common deregulated genes by both HDAC inhibitors inhibitors for $20 \mathrm{~h}$ and cell viability to bortezomib was measured. d Cell cycle profiles of SEM-resistant cells treated with DMSO, LBH589 $(5 \mathrm{nM})$, or SAHA $(2 \mu \mathrm{M})$ for $20 \mathrm{~h}$. Venn diagram (e) and heatmap of the overlapped 405 genes (f) of SEM parental and resistant cells treated with DMSO, LBH589 $(5 \mathrm{nM})$, or SAHA $(2 \mu \mathrm{M})$ for $20 \mathrm{~h}$. g GSEA analysis of datasets obtained from SEM-resistant cells after the treatment of DMSO, LBH589 $(5 \mathrm{nM})$, or SAHA $(2 \mu \mathrm{M}) .{ }^{*} P<0.05$; $* * P<0.01$; two-tailed $t$-test. Data represent the means of triplicate reactions $\pm \mathrm{SD}$.

in RS4;11 and SEM-resistant cells (Fig. S5A). Among them, 1323 common upregulated genes and 425 common downregulated genes were overlapped in HDAC inhibitortreated RS4;11 and SEM-resistant cells (Fig. S5B). Further analysis showed the overlap of DEGs involved in multiple biological processes, of which the cell proliferation pathway was among the top enriched terms (Fig. S5C, D). These results reveal that under HDAC inhibitor treatment, a large number of genes in different MLL-resistant cells were commonly induced, involving in several biological processes such as cell proliferation. Using GSEA, we 
A

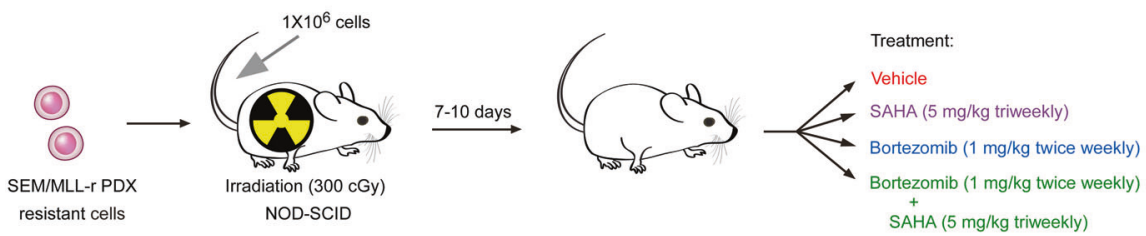

B

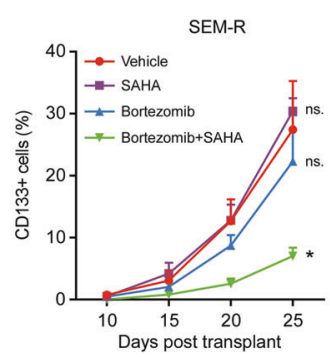

E

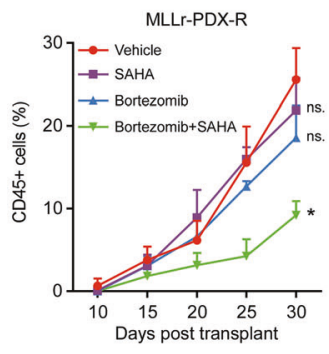

C

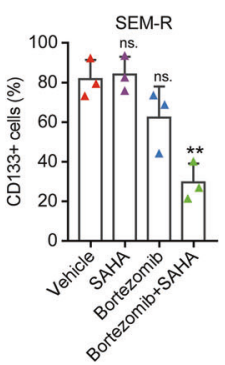

F

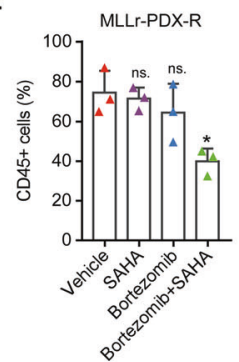

D

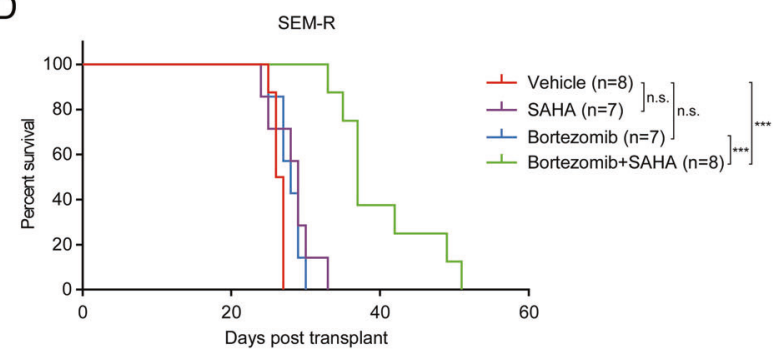

G

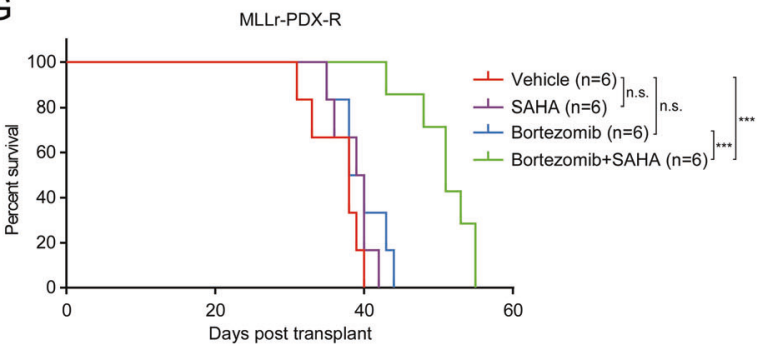

$\mathrm{H}$

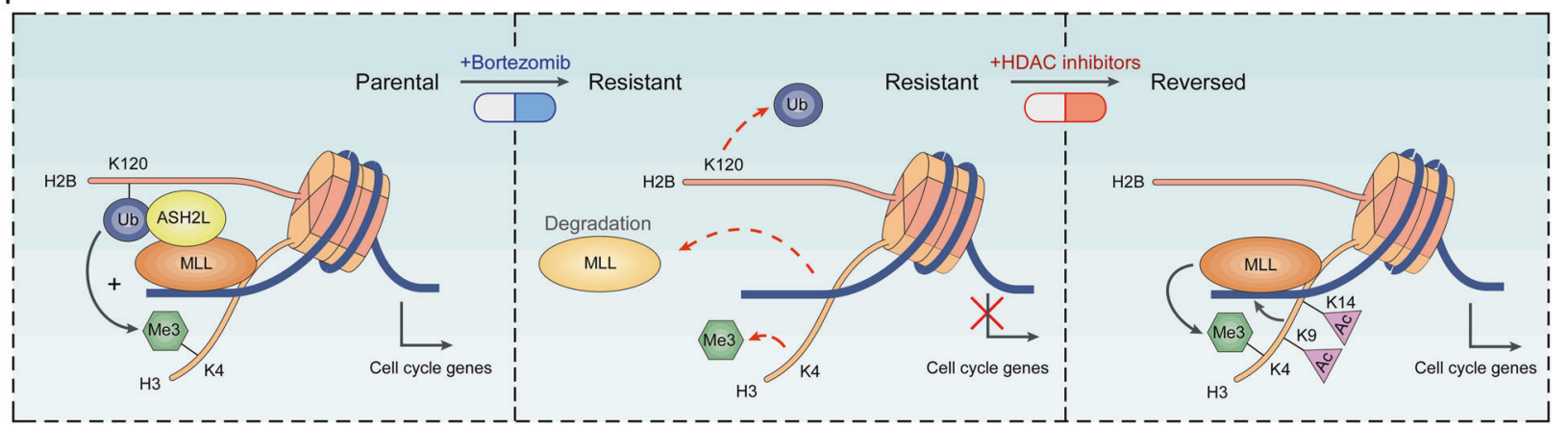

Fig. 7 The combination of PI and HDAC inhibitor overcomes PI resistance in vivo. a Treatment schedule for administration of bortezomib, SAHA, or vehicle. b, $\mathbf{c}$ Analysis of the recipient mice transplanted with SEM-resistant cells. The percentage of leukemic cells in the peripheral blood (b) or bone marrow (c) of the indicated groups of mice was monitored using CD133 antibody. The bone marrow cells from xenograft mice were collected 25 days post transplant. d Kaplan-Meier survival curves for the SEM resistance model from enrollment time. $\mathbf{e}, \mathbf{f}$ Analysis of the recipient mice transplanted with PI-resistant MLL-r PDX cells. The percentage of leukemic cells in the

established that the genes associated with cell cycle processes were significantly enriched in the treatment sets that were upregulated by HDAC inhibitors (Fig. 6g, Table S6), indicating that HDAC inhibitors restored the expression patterns of cell cycle genes. Collectively, these findings raise the possibility that a combinational therapy strategy using PIs and HDAC inhibitors may be effective in treating MLL-resistant cells. peripheral blood (e) or bone marrow (f) of the indicated groups of mice was monitored using CD45 antibody. The bone marrow cells from xenograft mice were collected 30 days post transplant. $\mathbf{g}$ Kaplan-Meier survival curves for the MLL-r PDX resistance model from enrollment time. $\mathbf{h}$ The illustration depicts how proteasome inhibition induces resistant cells and the therapeutic function of HDAC inhibitors in PI resistance. n.s. not significant; $* P<0.05 ; * * P<0.01$; $* * * P<0.001$; by two-tailed $t$-test or $\log$-rank test for significance. Data represent the means of triplicate reactions \pm SD.

\section{The combination of PI and HDAC inhibitor overcomes PI resistance in vivo}

Next, we transplanted the PI-resistant SEM cells into NODSCID mice and evaluated the in vivo efficacy of bortezomib alone or combination with SAHA [35] (Fig. 7a). Bortezomib single-agent treatment showed no benefit on these engrafted mice, consistent with the idea that these cells were 
refractory to bortezomib treatment. In contrast, mice treated with bortezomib in combination with SAHA showed notable responsiveness (Fig. 7b, c) and had a significant improvement in overall survival (Fig. 7d). We further performed the in vivo efficacy detection of combined therapy using MLL-r PDX cells. The monitored peripheral blood and bone marrow results showed that bortezomib in combination with SAHA significantly suppressed the progression of MLL leukemia (Fig. 7e, f). Moreover, compared with bortezomib or SAHA monotherapy, combined therapy significantly increased the overall survival of xenograft mice (Fig. 7g). Furthermore, immunoblot results showed that SAHA monotherapy significantly induced H3K9/14ac, chromatin-bound MLL and H3K4me3 in PI-resistant SEM cells and MLL-r PDX cells (Fig. S6A), while the induction of combined treatment was more obvious, accompanied by significant apoptosis (Fig. S6A, B), indicating that HDAC inhibitors can induce $\mathrm{H} 3 \mathrm{~K} 9 / 14 \mathrm{ac}$ and lead to the accumulation of MLL on chromatin to restore PI resistance. These results strongly suggest that a combinational therapy strategy using PIs and HDAC inhibitors, as opposed to sustained PI monotherapy, may be more effective in treating MLL leukemias by preventing the emergence of resistant cancer cells.

\section{Discussion}

The relatively rapid acquisition of drug resistance to cancer therapies remains a crucial obstacle [36]. PIs have dramatically improved the treatment of multiple myeloma (MM) and other hematological malignancies, but relapses are frequent, and acquired resistance to treatment eventually emerges [37, 38]. Despite many mechanisms that have been proposed for PI resistance in MM, how PI resistance inevitably develops in MLL leukemia remains elusive. Here, we provide a unique mechanism underlying drug resistance in MLL leukemia and the efficacy of combination therapy. We show evidence that MLL is indispensable for latent tumor suppression-mediated vulnerability of MLL leukemic cells to PI treatment. Our study highlights the importance of preventing the emergence of treatmentinduced drug-resistant cancer cells in cancer therapy. Conceivably, attenuating the acquisition of drug resistance would be an effective way to prevent treatment failure and relapse. To this end, combination and intermittent therapy strategies, as opposed to sustained monotherapy, should be utilized to prevent cancer cells from adapting a drugresistant state [39, 40].

HDAC inhibitors have potential therapeutic effects on cancers and can be used in combination with a variety of chemotherapies and immunotherapies, and the synergy between bortezomib and HDAC inhibitors has been reported in MM and other hematological malignancy cells [4143]. The drug interaction between bortezomib and HDAC inhibitors seems to be involving multiple mechanisms [44]. Nevertheless, our study suggests that preventing the emergence of resistant cancer cells may constitute a new rationale for this combinational therapy strategy. Histone acetylation induced by HDAC inhibitors can recruit wildtype MLL rather than MLL fusions on chromatin, thereby restoring MLL function and promoting cell cycle gene expression. These findings suggest that targeting MLL dysfunction by HDAC inhibition can be a promising strategy for treating the aggressive resistance in MLL leukemia. Of note, the combination of PI and HDAC inhibitor is being evaluated in a recent clinical trial of MLL-rearranged leukemia (NCT02553460), which demonstrated the promising efficacy of this combinational therapy strategy and the high clinical relevance of our study [20].

The discrepancy in p27 activation between acute and chronic PI treatment suggests that cell cycle deregulation in PI-resistant cells is achieved through an alternative mechanism. In our RNA-Seq data, chronic-induced dysregulation of many cell cycle genes, including cyclins, cyclindependent kinases (CDKs), and CDK inhibitors (CDKIs), which interact and influence each other in regulating cell cycle, were remarkably enriched and among the most significantly downregulated DEGs. This conflicting expression pattern of cell cycle genes suggesting that the cell cycle of PI-resistant MLL cells is in an inactive state and the expression level of p27 does not independently determine the cell cycle transition. Many observations suggest that p27 expression in MEFs and a few human cell lines is not completely associated with G1 arrest [45]. In addition, low levels of p27 observed in human tumors do not correlate with the proliferation index [46]. Our study demonstrated that the sensitivity of MLL leukemia cells to PI was related to the activation state of p27, and PI resistance was caused by a low replication state and the failure of p27 induction. Furthermore, we found that HDAC inhibitors not only induced the expression of p27 and other cell cycle genes, but also restored PI-induced p27 activation and thus restored cell sensitivity, suggesting that PIs selectively kill MLL cells during the cell cycle progression, and induced expression of p27 is necessary for the anti-MLL leukemia activity. Nevertheless, while HDAC inhibitors restored p27 induction in PI-resistant leukemic cells, they failed to repress leukemia development along in mice, indicating that additional pathways are required for killing leukemic cells by PI through MLL-fusion protein. Besides, we know very little about how these different cyclins, CDKs, and CDKIs differentially regulate the quiescence of MLL leukemic cells. To conclude, we are only beginning to appreciate the 
underlying importance of p27 and other cell cycle genes in the context of PI resistance. Thus, how such intrinsically and practically contradictory p27 protein dominates MLL cell sensitivity needs further exploration.

An expanding body of the literature emphasizes the contribution of epigenetic reprogramming in the development of inevitable drug resistance [47, 48]. We revealed that chronic PI treatment-induced epigenetic reprogramming caused unfavorable chromatin accessibility for MLL, resulting in less MLL to be recruited onto the chromatin. Subsequently, the loss of MLL function led to the dysregulation of cell cycle and the emergence of cell resistance. It is well recognized that cancer cells under drug stress primarily achieve cell fitness through modulating the signal transduction pathways governing these cellular stress response, and epigenetic changes are usually considered as secondary events [49]. Our study demonstrated that drug-induced epigenetic reprogramming might function as a driver in the adaptation process under therapy stress. Further elucidation of both epigenetic chromatin modification and accessibility may open new avenues for therapeutic targeting, including epigenetic modulators.

We and other groups have previously observed that MLL-r B-ALL cells, rather than AML cells, displayed selective sensitivity to PIs but not AML [18, 19]. Unlike ALL cells, the conflicting observations after the depletion of wild-type MLL allele in AML models have been the subject of intense research. Interestingly, while our results imply that MLL is the main mediator of the observed effects of bortezomib on cell cycle, which partially mediates the effect of H2Bub depletion in MLL-rearranged ALL cells, previous reports showed that the depletion of RNF20 but not MLL leads to inhibition of cell proliferation in MLL-rearranged AML cells, suggesting the effect of H2Bub depletion in AML cells is not mediated by MLL $[13,50]$. This discrepancy indicated that the wild-type allele of MLL might play different roles in MLL-rearranged ALL and AML cells. Given the importance of discovering therapeutic targets in MLL-rearranged leukemia, further studies are needed to clarify these discrepancies.

In summary, we have demonstrated that MLL leukemic cells under PI treatment can generally and intrinsically acquire an MLL dysfunction-mediated cell cycle deregulation resistant state (Fig. 7h), which reveals the phenotype switching mechanism may extend beyond MLL-rearranged B-ALL leukemias. We also find that HDAC inhibitors can reverse PI resistance, which extends the therapeutic concept of MLL restoring. However, since MLL may play a different role in diverse cancer types, whether our proposed mechanism may extend to other cancers beyond MLLrearranged leukemia remains an interesting topic requiring further investigation.

\section{Materials and methods}

\section{Plasmid constructions}

FLAG-tagged fragments consisting of MLL amino acids 1394-2665 (MLL $\left.{ }^{\text {Inter }}\right), \quad$ 1394-1788 (PHD1-3-Bromo), 1394-1632 (PHD1-3), 1626-1987 (Bromo-PHD4), and 1626-1788 (Bromo) derived from wild-type MLL were inserted into eukaryotic expression vectors pCI-neo (Promega) for transient transfection assays. Transfection was performed according to the manufacturer's protocol using FuGENE (Promega).

\section{Reagents}

Bortezomib (Velcade), panobinostat (LBH589), and vorinostat (SAHA, MK0683) were obtained from Selleck Chemicals. The IRAK1/4 inhibitor was obtained from MedChemExpress. Cycloheximide was obtained from Sigma.

\section{Cell culture}

Human pro-B MLL leukemia cell lines RS4;11 and SEM [51] were purchased from DSMZ. Cells were cultured in Gibco RPMI 1640 containing $10 \%$ FBS at $37^{\circ} \mathrm{C}$ with $5 \%$ $\mathrm{CO}_{2}$ and were maintained between a density of $5 \times 10^{5}$ cells $/ \mathrm{mL}$ and $2 \times 10^{6}$ cells $/ \mathrm{mL}$. SEM or RS4;11 cells were continuously treated with a sublethal dose of bortezomib $(5 \mathrm{nM})$ for over 4 weeks. Cells were passaged and the inhibitor was replenished every 3 days. The half-maximal dosage effect $\left(\mathrm{IC}_{50}\right)$ values were measured intermittently until the drug resistance was acquired. After the drug resistance development, the "Resistant" cells were collected for analysis.

\section{Cell viability and cell proliferation assays}

The CellTiter 96 MTS assay (Promega) was used to determine the cytotoxicity of the relevant drugs and cell proliferation, according to the manufacturer's instructions. Cell viability was measured with MTS assay $24 \mathrm{~h}$ after the addition of bortezomib with graded concentrations in triplicates. Cells were pretreated with IRAK1/4 inhibitor for $48 \mathrm{~h}$ before the measurement.

\section{Apoptosis and cell cycle assays}

Apoptosis and cell cycle were measured using the PE Annexin V Apoptosis Detection Kit and APC BrdU Flow Kit from BD Pharmingen as described by the manufacturer, respectively. Cells staining with fluorochromes were acquired using flow cytometer and data were analyzed using FlowJo software. 


\section{shRNA-mediated knockdown}

Target sequences against human MLL C-terminus, ASH2L, RNF20, and a control scrambled sequence that has no significant homology with the human genome were inserted into the pLKO.1 vector, according to the manufacturer's protocol (Addgene). Generated lentivirus carrying shRNA was used to infect target cells for 2 days, and the cells were subjected to puromycin selection at $2 \mu \mathrm{g} / \mathrm{mL}$.

\section{RNA-Seq, ChIP, and qRT-PCR}

Total RNA was extracted from Trizol according to the manufacturer's instructions. The mRNA-seq library was sequenced using BGISEQ-500RS for 100-bp paired-end sequencing. After quality control, clean reads were aligned to the human genome (UCSC hg19) by Tophat2.1.0 with a maximum of two mismatches for each reads. Gene ontology analysis was performed using the KEGG database and DAVID (http://david.abcc.ncifcrf.gov) for pathway analysis. GSEA was executed using public software from the Broad Institute (http://software.broadinstitute.org/gsea). ChIP assays were performed following the described protocol [52]. Cellular RNA and precipitated DNA samples were reverse transcribed with random primers and detection was performed using 7500 Real-Time PCR Systems (Applied Biosystems). Primers used for qRT-PCR assays were listed in Table S7.

\section{Immunoblots}

Human CD133-PE antibody was obtained from Miltenyi Biotec. Human CD45-FITC antibody was obtained from Biolegend. Mouse antibody against p27 was obtained from BD Biosciences. Rabbit antibodies against $\mathrm{MLL}^{\mathrm{C} 180}$ (D6G8N), MLL ${ }^{\mathrm{N} 320}$ (D2M7U), ASH2L, CD133, UbiquitylHistone H2B (Lys120) (H2Bub), Tri-Methyl-Histone H3 (Lys4) (H3K4me3), Histone H3, and mouse anti-Histone H2B antibody were purchased from Cell Signaling Technology. Rabbit anti-RNF20 antibody was purchased from ABclonal Technology. Rabbit anti-Acetyl-Histone H3 (Lys9/14) (H3K9/14ac) antibody was obtained from Thermo Fisher Scientific. Mouse anti-FLAG, $\beta$-Actin, and Tubulin antibodies were obtained from Sigma Aldrich. Antibodies were detected using the enhanced chemiluminescence method (PerkinElmer). Immunoblot signals were acquired with the Amersham Imager 600 (General Electric Company).

\section{Peptide pull-down and chromatin association assays}

Peptide pull-down assays have been described previously [3]. In brief, individual biotin-conjugated histone $\mathrm{H} 3$ peptide (amino acids $1-21 ; 2 \mu \mathrm{g}$ ) or biotin-conjugated acetyl-histone H3 (Lys9/14) peptide (amino acids 1-21; $2 \mu \mathrm{g}$ ) (Millipore) were incubated with nuclear extracts, precipitated using streptavidin beads (GE Healthcare). The co-precipitated protein was detected by immunoblots. Chromatin-enriched fractions were purified as described previously [3].

\section{Mouse studies}

NOD-SCID mice were purchased from Vital River Laboratory. MLL-r PDX were generated from two MLLAF4-fusioned B-ALL patients. The characteristics of these MLL patients are detailed in Table S1. In total, $1 \times 10^{6}$ bone marrow cells were collected and intravenously injected into NOD-SCID mice (6-8 weeks, male or female). Mice were then administered bortezomib intravenously at $1 \mathrm{mg} / \mathrm{kg}$ on a twice-weekly schedule, or administered vehicle, beginning 7 days after the xenograft. The percentage of leukemic cells in the peripheral blood of mice was monitored using CD45 antibody. To evaluate the in vivo efficacy of bortezomib combined with SAHA, $1 \times 10^{6}$ SEM-resistant or MLL-r PDX-resistant cells were intravenously injected into NODSCID mice. Mice were then administered bortezomib intravenously at $1 \mathrm{mg} / \mathrm{kg}$ on a twice-weekly schedule, or administered SAHA intraperitoneally at $5 \mathrm{mg} / \mathrm{kg}$ three times per week, beginning 7 days after the xenograft. Mice undergoing bortezomib or SAHA monotherapy also received the vehicle. The percentage of leukemic cells in the peripheral blood of mice was monitored using CD133 and CD45 antibody, respectively [53]. Mice were sacrificed by inhalation of $\mathrm{CO}_{2}$ when they became moribund. For hematoxylin-eosin staining, the spleen and liver of mice were collected. Animal care and sacrifice were conducted according to methods approved by the Animal Care and Use Committee, the Center for Animal Experiments of Shanghai Jiao Tong University.

\section{Statistical analysis}

The Student's $t$-test was used to analyze the differences between the groups. Means were illustrated using a histogram with error bars representing \pm the standard deviation (SD), and statistical relevance was evaluated using the following $P$ values: $* P<0.05, * * P<0.01$, or $* * * P<0.001$.

\section{Data availability}

All data generated or analyzed during this study are included in the paper and its supplementary information files. RNA-seq data are available in the Gene Expression Omnibus (GEO) database under accession number GSE138177. 
Funding This work was supported by the National Key Research and Development Program of China (2018YFA0107802), the National Natural Science Foundation of China (81973996 and 81570119), the Shanghai Municipal Education Commission Gaofeng Clinical Medicine Grant (20161304), the Program of Shanghai Academic Research Leader (19XD1402500), the Shanghai Municipal Health Commission (2019CXJQ01), the Joint Funds for the Innovation of Science and Technology of Fujian Province of China (No.2017Y9005), the Collaborative Innovation Center of Hematology, and the Samuel Waxman Cancer Research Foundation.

Author contributions MG designed and performed most of the experiments, analyzed the data, and wrote the draft paper; DL, ZQ, and YS performed some experiments and data analyses; TK, SZ, and SW performed some experiments; $\mathrm{HX}$ and $\mathrm{CZ}$ provided expertise and extensively edited the paper; SS provided expertise on the in vivo experiments; HL and ZX contributed grant support, designed the entire project, and wrote the paper. All authors discussed the results and commented on the paper.

\section{Compliance with ethical standards}

Conflict of interest The authors declare that they have no conflict of interest.

Ethical approval The study procedures were approved by the Ethics Committee of Shanghai Children's Medical Center, Shanghai Jiao Tong University School of Medicine, and received the written informed consent of each patient. The study was performed in accordance with the Declaration of Helsinki.

Publisher's note Springer Nature remains neutral with regard to jurisdictional claims in published maps and institutional affiliations.

Open Access This article is licensed under a Creative Commons Attribution 4.0 International License, which permits use, sharing, adaptation, distribution and reproduction in any medium or format, as long as you give appropriate credit to the original author(s) and the source, provide a link to the Creative Commons license, and indicate if changes were made. The images or other third party material in this article are included in the article's Creative Commons license, unless indicated otherwise in a credit line to the material. If material is not included in the article's Creative Commons license and your intended use is not permitted by statutory regulation or exceeds the permitted use, you will need to obtain permission directly from the copyright holder. To view a copy of this license, visit http://creativecommons. org/licenses/by/4.0/.

\section{References}

1. Shilatifard A. The COMPASS family of histone H3K4 methylases: mechanisms of regulation in development and disease pathogenesis. Annu Rev Biochem. 2012;81:65-95.

2. Takeda S, Chen DY, Westergard TD, Fisher JK, Rubens JA, Sasagawa S, et al. Proteolysis of MLL family proteins is essential for taspase1-orchestrated cell cycle progression. Genes Dev. 2006;20:2397-409.

3. Liu H, Takeda S, Kumar R, Westergard TD, Brown EJ, Pandita TK, et al. Phosphorylation of MLL by ATR is required for execution of mammalian S-phase checkpoint. Nature. 2010;467:343-6.

4. Hsieh JJ, Cheng EH, Korsmeyer SJ. Taspase1: a threonine aspartase required for cleavage of MLL and proper HOX gene expression. Cell. 2003;115:293-303.
5. Krivtsov AV, Armstrong SA. MLL translocations, histone modifications and leukaemia stem-cell development. Nat Rev Cancer. 2007;7:823-33.

6. Meyer C, Burmeister T, Groger D, Tsaur G, Fechina L, Renneville A, et al. The MLL recombinome of acute leukemias in 2017. Leukemia. 2018;32:273-84.

7. Thiel AT, Blessington P, Zou T, Feather D, Wu X, Yan J, et al. MLL-AF9-induced leukemogenesis requires coexpression of the wild-type Mll allele. Cancer Cell. 2010;17:148-59.

8. Milne TA, Kim J, Wang GG, Stadler SC, Basrur V, Whitcomb SJ, et al. Multiple interactions recruit MLL1 and MLL1 fusion proteins to the HOXA9 locus in leukemogenesis. Mol Cell. 2010;38:853-63.

9. Cao F, Townsend EC, Karatas H, Xu J, Li L, Lee S, et al. Targeting MLL1 H3K4 methyltransferase activity in mixed-lineage leukemia. Mol Cell. 2014;53:247-61.

10. Xu H, Valerio DG, Eisold ME, Sinha A, Koche RP, Hu W, et al. NUP98 fusion proteins interact with the NSL and MLL1 complexes to drive leukemogenesis. Cancer Cell. 2016;30:863-78.

11. Riedel SS, Haladyna JN, Bezzant M, Stevens B, Pollyea DA, Sinha AU, et al. MLL1 and DOT1L cooperate with meningioma-1 to induce acute myeloid leukemia. J Clin Investig. 2016;126:1438-50.

12. Mishra BP, Zaffuto KM, Artinger EL, Org T, Mikkola HK, Cheng $\mathrm{C}$, et al. The histone methyltransferase activity of MLL1 is dispensable for hematopoiesis and leukemogenesis. Cell Rep. 2014;7:1239-47.

13. Chen Y, Anastassiadis K, Kranz A, Stewart AF, Arndt K, Waskow C, et al. MLL2, not MLL1, plays a major role in sustaining MLL-rearranged acute myeloid leukemia. Cancer Cell. 2017;31:755-70.e6.

14. Liang K, Volk AG, Haug JS, Marshall SA, Woodfin AR, Bartom ET, et al. Therapeutic targeting of MLL degradation pathways in MLL-rearranged leukemia. Cell. 2017;168:59.

15. Zhao Z, Wang L, Volk AG, Birch NW, Stoltz KL, Bartom ET, et al. Regulation of MLL/COMPASS stability through its proteolytic cleavage by taspase 1 as a possible approach for clinical therapy of leukemia. Genes Dev. 2019;33:61-74.

16. Muntean AG, Hess JL. The pathogenesis of mixed-lineage leukemia. Annu Rev Pathol. 2012;7:283-301.

17. Ahmad K, Katryniok C, Scholz B, Merkens J, Loscher D, Marschalek R, et al. Inhibition of class I HDACs abrogates the dominant effect of MLL-AF4 by activation of wild-type MLL. Oncogenesis. 2014;3:e127.

18. Liu H, Westergard TD, Cashen A, Piwnicaworms DR, Kunkle L, Vij R, et al. Proteasome inhibitors evoke latent tumor suppression programs in Pro-B MLL leukemias through MLL-AF4. Cancer Cell. 2014;25:530.

19. Rushworth SA, Bowles KM, MacEwan DJ. High basal nuclear levels of Nrf2 in acute myeloid leukemia reduces sensitivity to proteasome inhibitors. Cancer Res. 2011;71:1999-2009.

20. Koss C, Nance S, Connelly M, Ma J, Shelat A, Cotton A, et al. Targeted inhibition of the MLL transcriptional complex by proteosome inhibitors elicits a high response rate in relapsed/refractory MLL rearranged leukemia. Blood. 2014;124:972.

21. Milne TA, Hughes CM, Lloyd R, Yang Z, Rozenblatt-Rosen O, Dou Y, et al. Menin and MLL cooperatively regulate expression of cyclin-dependent kinase inhibitors. Proc Natl Acad Sci USA. 2005;102:749-54.

22. Xia ZB, Popovic R, Chen J, Theisler C, Stuart T, Santillan DA, et al. The MLL fusion gene, MLL-AF4, regulates cyclindependent kinase inhibitor CDKN1B (p27kip1) expression. Proc Natl Acad Sci USA. 2005;102:14028-33.

23. Wainwright EN, Scaffidi P. Epigenetics and cancer stem cells: unleashing, hijacking, and restricting cellular plasticity. Trends Cancer. 2017;3:372-86. 
24. Prenzel T, Begus-Nahrmann Y, Kramer F, Hennion M, Hsu C, Gorsler T, et al. Estrogen-dependent gene transcription in human breast cancer cells relies upon proteasome-dependent monoubiquitination of histone H2B. Cancer Res. 2011;71:5739-53.

25. Espinosa JM. Histone H2B ubiquitination: the cancer connection. Genes Dev. 2008;22:2743-9.

26. Rao RC, Dou Y. Hijacked in cancer: the KMT2 (MLL) family of methyltransferases. Nat Rev Cancer. 2015;15:334-46.

27. Wu L, Lee SY, Zhou B, Nguyen UT, Muir TW, Tan S, et al. ASH2L regulates ubiquitylation signaling to MLL: transregulation of $\mathrm{H} 3 \mathrm{~K} 4$ methylation in higher eukaryotes. Mol Cell. 2013;49:1108-20.

28. Lee JS, Shukla A, Schneider J, Swanson SK, Washburn MP, Florens L, et al. Histone crosstalk between H2B monoubiquitination and $\mathrm{H} 3$ methylation mediated by COMPASS. Cell. 2007;131:1084-96.

29. Allen MD, Grummitt CG, Hilcenko C, Min SY, Tonkin LM, Johnson CM, et al. Solution structure of the nonmethyl-CpGbinding CXXC domain of the leukaemia-associated MLL histone methyltransferase. EMBO J. 2006;25:4503-12.

30. Wang Z, Song J, Milne TA, Wang GG, Li H, Allis CD, et al. Pro isomerization in MLL1 PHD3-bromo cassette connects H3K4me readout to $\mathrm{CyP} 33$ and $\mathrm{HDAC}-$ mediated repression. Cell. 2010;141:1183-94.

31. Mujtaba S, Zeng L, Zhou MM. Structure and acetyl-lysine recognition of the bromodomain. Oncogene. 2007;26:5521-7.

32. Nakanishi S, Sanderson BW, Delventhal KM, Bradford WD, Staehling-Hampton K, Shilatifard A. A comprehensive library of histone mutants identifies nucleosomal residues required for H3K4 methylation. Nat Struct Mol Biol. 2008;15:881-8.

33. West AC, Johnstone RW. New and emerging HDAC inhibitors for cancer treatment. J Clin Investig. 2014;124:30-9.

34. San-Miguel JF, Hungria VT, Yoon SS, Beksac M, Dimopoulos MA, Elghandour A, et al. Panobinostat plus bortezomib and dexamethasone versus placebo plus bortezomib and dexamethasone in patients with relapsed or relapsed and refractory multiple myeloma: a multicentre, randomised, double-blind phase 3 trial. Lancet Oncol. 2014;15:1195-206.

35. Mazur PK, Herner A, Mello SS, Wirth M, Hausmann S, SanchezRivera FJ, et al. Combined inhibition of BET family proteins and histone deacetylases as a potential epigenetics-based therapy for pancreatic ductal adenocarcinoma. Nat Med. 2015;21:1163-71.

36. Glickman MS, Sawyers CL. Converting cancer therapies into cures: lessons from infectious diseases. Cell. 2012;148:1089-98.

37. Manasanch EE, Orlowski RZ. Proteasome inhibitors in cancer therapy. Nat Rev Clin Oncol. 2017;14:417-33.

38. Moreau P, Richardson PG, Cavo M, Orlowski RZ, San Miguel JF, Palumbo A, et al. Proteasome inhibitors in multiple myeloma: 10 years later. Blood. 2012;120:947-59.
39. Goldman A, Majumder B, Dhawan A, Ravi S, Goldman D, Kohandel $\mathrm{M}$, et al. Temporally sequenced anticancer drugs overcome adaptive resistance by targeting a vulnerable chemotherapy-induced phenotypic transition. Nat Commun. 2015;6:6139.

40. Salgia R, Kulkarni P. The genetic/non-genetic duality of drug 'resistance' in cancer. Trends Cancer. 2018;4:110-8.

41. Pei XY, Dai Y, Grant S. Synergistic induction of oxidative injury and apoptosis in human multiple myeloma cells by the proteasome inhibitor bortezomib and histone deacetylase inhibitors. Clin Cancer Res. 2004;10:3839-52.

42. Hideshima T, Bradner JE, Wong J, Chauhan D, Richardson P, Schreiber SL, et al. Small-molecule inhibition of proteasome and aggresome function induces synergistic antitumor activity in multiple myeloma. Proc Natl Acad Sci USA. 2005;102:8567-72.

43. Zhang QL, Wang L, Zhang YW, Jiang XX, Yang F, Wu WL, et al. The proteasome inhibitor bortezomib interacts synergistically with the histone deacetylase inhibitor suberoylanilide hydroxamic acid to induce T-leukemia/lymphoma cells apoptosis. Leukemia. 2009;23:1507-14.

44. McConkey D. Proteasome and HDAC: who's zooming who? Blood. 2010;116:308-9.

45. Kaldis P. Another piece of the p27Kip1 puzzle. Cell. 2007;128:241-4.

46. Loda M, Cukor B, Tam SW, Lavin P, Fiorentino M, Draetta GF, et al. Increased proteasome-dependent degradation of the cyclindependent kinase inhibitor p27 in aggressive colorectal carcinomas. Nat Med. 1997;3:231-4.

47. Shaffer SM, Dunagin MC, Torborg SR, Torre EA, Emert B, Krepler C, et al. Rare cell variability and drug-induced reprogramming as a mode of cancer drug resistance. Nature. 2017;546:431-5.

48. Liau BB, Sievers C, Donohue LK, Gillespie SM, Flavahan WA, Miller TE, et al. Adaptive chromatin remodeling drives glioblastoma stem cell plasticity and drug tolerance. Cell Stem Cell. 2017;20:233-46.e7.

49. Flavahan WA, Gaskell E, Bernstein BE. Epigenetic plasticity and the hallmarks of cancer. Science. 2017;357:eaal2380.

50. Wang E, Kawaoka S, Yu M, Shi J, Ni T, Yang W, et al. Histone $\mathrm{H} 2 \mathrm{~B}$ ubiquitin ligase RNF20 is required for MLL-rearranged leukemia. Proc Natl Acad Sci USA. 2013;110:3901-6.

51. Drexler HG, Quentmeier H, Macleod RA. Malignant hematopoietic cell lines: in vitro models for the study of MLL gene alterations. Leukemia. 2004;18:227-32.

52. Weinmann AS, Farnham PJ. Identification of unknown target genes of human transcription factors using chromatin immunoprecipitation. Methods. 2002;26:37-47.

53. Li D, Hu Y, Jin Z, Zhai Y, Tan Y, Sun Y, et al. TanCAR T cells targeting CD19 and CD133 efficiently eliminate MLL leukemic cells. Leukemia. 2018;32:2012-6. 